\title{
AN ALTERNATIVE REPARAMETRIZATION FOR THE WEIGHTED LINDLEY DISTRIBUTION
}

\author{
Josmar Mazucheli $^{1 *}$, Emílio Augusto Coelho-Barros ${ }^{2}$ \\ and Jorge Alberto Achcar ${ }^{3}$
}

Received February 19, 2015 / Accepted June 4, 2016

\begin{abstract}
Recently, [12] introduced a generalization of a one parameter Lindley distribution and named it as a weighted Lindley distribution. Considering this new introduced weighted Lindley distribution, we propose a reparametrization on the shape parameter leading it to be orthogonal to the other shape parameter. In this alternative parametrization, we get a direct interpretation for this transformed parameter which is the mean survival time. For illustrative purposes, the weighted Lindley distribution on the new parametrization is applied on two real data sets. The one parameter Lindley distribution and its generalized form are fitted for the considered data sets.
\end{abstract}

Keywords: generalized Lindley distribution, Lindley distribution, orthogonal parameters, survival analysis, weighted Lindley distribution.

\section{INTRODUCTION}

A non negative random variable $T$ follows the two-parameter weighted Lindley distribution, [12], with shape parameters $\mu>0$ and $\beta>0$ if its probability density function is given by:

$$
f(t \mid \mu, \beta)=\frac{\mu^{\beta+1}}{(\mu+\beta) \Gamma(\beta)} t^{\beta-1}(1+t) e^{-\mu t},
$$

where $t>0$ and $\Gamma(\beta)=\int_{0}^{\infty} t^{\beta-1} e^{-t} d t$ is the gamma function. From (1), the corresponding survival and hazard functions, are given, respectively, by:

$$
S(t \mid \mu, \beta)=\frac{(\mu+\beta) \Gamma(\beta, \mu t)+(\mu t)^{\beta} e^{-\mu t}}{(\mu+\beta) \Gamma(\beta)},
$$

*Corresponding author.

${ }^{1}$ Universidade Estadual de Maringá, Departamento de Estatística, Maringá, PR, Brasil. E-mail: jmazucheli@ gmail.com

${ }^{2}$ Universidade Tecnológica Federal do Paraná, Departamento Acadêmico de Matemática, Cornélio Procópio, PR, Brasil. E-mail: eabarros@utfpr.edu.br

${ }^{3}$ Universidade de São Paulo, Departamento de Medicina Social, Ribeirão Preto, SP, Brasil. E-mail: achcar@ fmrp.usp.br 
and

$$
h(t \mid \mu, \beta)=\frac{\mu^{\beta+1} t^{\beta-1}(1+t) e^{-\mu t}}{(\mu+\beta) \Gamma(\beta, \mu t)+(\mu t)^{\beta} e^{-\mu t}},
$$

where $\Gamma(a, b), a>0$ and $b \geq 0$, is the upper incomplete gamma function (see, [29]), defined as $\int_{b}^{\infty} t^{a-1} e^{-t} d t$

In (1), taking the shape parameter $\beta=1$ we have the one parameter Lindley distribution as a special case. The one parameter Lindley distribution was introduced by Lindley (see, Lindley 1958 and 1965) as a new distribution useful to analyze lifetime data, especially in applications modeling stress-strength reliability. [13] studied the properties of the one parameter Lindley distribution under a careful mathematical approach. These authors also showed, in a numerical example, that the Lindley distribution usually gives better fit for the data when compared to the standard Exponential distribution. A generalized Lindley distribution, which includes as special cases the Exponential and Gamma distributions was introduced by [36]. Ghitany and Al-Mutari (2008) considered a size-biased Poisson-Lindley distribution and [31] introduced the Poisson-Lindley distribution to model count data. Some properties of the Poisson-Lindley distribution, its derived distributions and some mixtures of this distribution were studied by [5, 6, 24]. A zero-truncated Poisson-Lindley was considered in [10]. A study on the inflated PoissonLindley distribution was presented in [7] and the Negative Binomial-Lindley distribution was introduced in [37]. The one parameter Lindley distribution in the competing risks scenario was considered in [26].

Since the standard one parameter Lindley distribution does not provide enough flexibility to analyze different types of lifetime data, the two-parameter weighted Lindley distribution could be a good alternative in the analysis of lifetime data. A nice feature of the two-parameter weighted Lindley distribution is that its hazard function has a bathtub form for $0<\beta<1$ and it is increasing for $\beta \geq 1$, for all $\mu>0$.

It is important to point out, that in the last years, several distributions have been introduced in the literature to model bathtub hazard functions but in general these distributions have three or more parameters usually depending on numerical methods to find the maximum likelihood estimates which could be, in general, not very accurate. In this case good reparametrizations with less parameters could be very useful in applications. A comprehensive review of the existing know distributions that exhibit bathtub shape is provided in [30, 17, 3, 28]. In addition to the weighted Lindley distribution, that can be used to model bathtub-shaped failure rate, we also could consider as alternatives, four other two-parameter distributions introduced in the literature $[18,8,15,35]$ with this behavior.

The main goal of this paper is to propose an alternative parametrization for the one shape parameter of the weighted Lindley distribution. In the proposed parametrization, we get the new parameter orthogonal to the other shape parameter where this new reparametrized form of the parameter gives the mean survival time. The obtained orthogonality of the reparametrized form of the parameter is related to the observed Fisher information [2]. Orthogonal parameters have many advantages in the inference results as, for example, for large sample sizes we have independence among the maximum likelihood of the orthogonal parameters, since the Fisher information 
matrix is diagonal. Other advantage of orthogonal parameters is related to the conditional likelihood approach (for further details see, Cox \& Reid, 1987; Lancaster, 2002; Louzada-Neto \& Pardo-Fernandez, 2001; Louzada \& Cavali, 2014).

The paper is organized as follows. In Section 2 the likelihood function for the two-parameter weighted Lindley distribution is formulated where we also present the proposed orthogonal reparametrization. Two examples considering real data sets are provided in Section 3 where its observed that the weighted Lindley distribution gives better fit for the data when compared to the one-parameter Lindley distribution and the generalized Lindley distribution. Some conclusions are presented in Section 4.

\section{THE LIKELIHOOD FUNCTION}

Let $\mathbf{t}=\left(t_{1}, \ldots, t_{n}\right)$ be a realization of the random sample $\mathbf{T}=\left(T_{1}, \ldots, T_{n}\right)$, where $T_{1}, \ldots, T_{n}$ are i.i.d. (identically independent distribution) random variables according to a two-parameter Lindley distribution, with shape parameters $\mu>0$ and $\beta>0$. From (1) the likelihood function can be written as:

$$
L(\mu, \beta \mid \mathbf{t})=\left(\frac{\mu^{\beta+1}}{(\mu+\beta) \Gamma(\beta)}\right)^{n} e^{-\mu T_{0}} \prod_{i=1}^{n} t_{i}^{\beta-1}\left(1+t_{i}\right),
$$

where $T_{0}=\sum_{i=1}^{n} t_{i}$ and $\Gamma(\beta)=\int_{0}^{\infty} y^{\beta-1} e^{-y} d y$ is the gamma function. From (4), the loglikelihood function for $\mu$ and $\beta, l(\mu, \beta \mid \mathbf{t})$, is given by:

$$
l(\alpha, \beta \mid \mathbf{t})=n[(\beta+1) \log (\mu)-\log (\mu+\beta)-\log \Gamma(\beta)]-\mu T_{0}+(\beta-1) T_{1}+T_{2},
$$

where $T_{1}=\sum_{i=1}^{n} \log \left(t_{i}\right)$ and $T_{2}=\sum_{i=1}^{n} \log \left(1+t_{i}\right)$.

Differentiating (5) with respect to $\mu$ and $\beta$ and setting the results equal to zero we have:

$$
\begin{aligned}
\frac{\partial}{\partial \mu} l(\mu, \beta \mid \mathbf{t}) & =n\left[\frac{\beta+1}{\mu}-\frac{1}{(\mu+\beta)}\right]-T_{0}=0 \\
\frac{\partial}{\partial \beta} l(\mu, \beta \mid \mathbf{t}) & =n\left[\log (\mu)-\frac{1}{(\mu+\beta)}-\psi(\beta)\right]+T_{1}=0,
\end{aligned}
$$

where $\psi(\beta)=\frac{d}{d \beta} \log \Gamma(\beta)$ is the digamma function. The maximum likelihood estimates, $\hat{\mu}$ and $\hat{\beta}$, for $\mu$ and $\beta$, respectively, are obtained by solving equations (6) and (7) in $\mu$ and $\beta$, respectively.

From (6), the maximum likelihood estimate for $\mu$ is obtained as a function of $\beta, \hat{\mu}(\beta)$, given by:

$$
\hat{\mu}(\beta)=\frac{\beta\left(n-T_{0}\right)+\sqrt{\beta^{2}\left(n+T_{0}\right)^{2}+4 n \beta T_{0}}}{2 T_{0}} .
$$

In this way, replace $\mu$ in (7) by $\hat{\mu}(\beta)$ given by (8), which leads to an equation with only one variable $\beta$. After choose an initial value for $\beta$, use standard Newton-Raphson algorithm to find 
the maximum likelihood estimator for $\beta$. With the obtained maximum likelihood estimator for $\beta$ get the maximum likelihood estimator for $\mu$ using equation (8).

Based on a single observation, the observed information matrix, $I(\mu, \beta)$, is given by:

$$
I(\mu, \beta)=\left(\begin{array}{cc}
\frac{\beta+1}{\mu^{2}}-\frac{1}{(\mu+\beta)^{2}} & -\left[\frac{1}{\mu}+\frac{1}{(\mu+\beta)^{2}}\right] \\
-\left[\frac{1}{\mu}+\frac{1}{(\mu+\beta)^{2}}\right] & \psi^{\prime}(\beta)-\frac{1}{(\mu+\beta)^{2}}
\end{array}\right),
$$

where $\psi^{\prime}(\beta)=\frac{d^{2}}{d \beta^{2}} \log \Gamma(\beta)$, and the terms in the $(2 \times 2)$ observed Fisher information matrix (9) are obtained from the second derivatives given by,

$$
I_{11}(\mu, \beta)=-\frac{\partial^{2}}{\partial \mu^{2}} l(\mu, \beta \mid \mathbf{t}), \quad I_{22}(\mu, \beta)=-\frac{\partial^{2}}{\partial \beta^{2}} l(\mu, \beta \mid \mathbf{t})
$$

and

$$
I_{12}(\mu, \beta)=-\frac{\partial^{2}}{\partial \mu \partial \beta} l(\mu, \beta \mid \mathbf{t}) .
$$

The maximum likelihood estimates for $\mu$ and $\beta$ have asymptotic bivariate normal distribution with mean $(\mu, \beta)$ and variance-covariance matrix given by the inverse of the Fisher Information matrix (9) locally at the maximum likelihood estimates $\hat{\mu}$ and $\hat{\beta}$. Since the data is independent, the information matrix (9) is equal to the expected information matrix.

In this paper we propose to reparametrize the two-parameter weighted Lindley distribution such that $(\mu, \beta)$ is transformed to $(\theta, \beta)$, where:

$$
\theta=g(\mu, \beta)=\frac{\beta(\mu+\beta+1)}{\mu(\mu+\beta)}
$$

where $\theta>0$ is the mean of the weighted Lindley distribution with parameters $\mu$ and $\beta$ and

$$
g^{-1}(\theta)=\mu=\frac{\beta(1-\theta)+\sqrt{\beta^{2}(\theta-1)^{2}+4 \theta \beta(\beta+1)}}{2 \theta} .
$$

Using the construction method of orthogonality parameters, proposed in [9], and from (9) we observe that $\mu$ is obtained as solution of the following orthogonality differential equation:

$$
\overbrace{\left[\frac{\beta+1}{\mu^{2}}-\frac{1}{(\mu+\beta)^{2}}\right]}^{I_{11}(\mu, \beta)} \frac{\partial \mu}{\partial \beta}-\underbrace{\left[\frac{1}{\mu}+\frac{1}{(\mu+\beta)^{2}}\right]}_{I_{12}(\mu, \beta)}=0
$$

In this new parametrization we have that the maximum likelihood estimate for $\theta$ is given by $\hat{\theta}=n^{-1} \sum_{i=1}^{n} t_{i}$ and $\operatorname{Cov}(\hat{\theta}, \hat{\beta})=0$. The orthogonality between $\theta$ and $\beta$ implies that the information matrix is asymptotically diagonal which implies that the the maximum likelihood estimates $\hat{\theta}$ and $\hat{\beta}$ are asymptotically independent. The orthogonality simplify the parameters estimation process and its interpretation. For the weighted Lindley distribution the parameter interpretation in the orthogonal parametrization is obvious since $\hat{\theta}$ is the mean time to failure. Further orthogonality consequences are pointed out in [9]. 


\section{APPLICATIONS}

In this section we fit the two-parameter weighted Lindley distribution (WL) to two real data sets. For comparative purposes we also have considered two alternative models: $(\mathrm{L})$ : the one parameter Lindley distribution, $f(t \mid \mu)=\frac{\mu^{2}}{\mu+1}(1+t) e^{-\mu t}$, and (GL): the generalized Lindley distribution, $f(t \mid \mu, \beta, \gamma)=\frac{\mu^{\beta+1}}{(\mu+\gamma) \Gamma(\beta+1)}(\beta+\gamma t) t^{\beta-1} e^{-\mu t},[36]$.

The first data set was reported by [4], and employed by [14] among others, represents the survival times (in days) of 72 guinea pigs infected with virulent tubercle bacilli, regimen 4.3. The regimen number is the common log of the number of bacillary units in $0.5 \mathrm{ml}$ of challenge solution. The second data set was extracted from [33], see also [25], representing hours to failure of 59 test conductors of 400-micrometer length. All specimens ran to failure at a certain high temperature and current density. The 59 specimens were all tested under the same temperature and current density.

Table 1 list for the two data sets and models L, WL and GL the maximum likelihood estimates and their standard errors. For comparative purposes the estimates are also presented in the original parameterization and were obtained using SAS/NLMIXED procedure, [32], by applying the Newton-Raphson algorithm. For the WL model, in the orthogonal parameterization, we have $\widehat{\theta}=\bar{t}=176.82$ (data set 1 ) and $\widehat{\theta}=\bar{t}=6.98$ (data set 2 ). The standard errors are given, respectively, by 11.86 and 0.21 .

Table 1 - Maximum likelihood (standard error) estimates for Lindley (L), weighted Lindley (WL) and generalized Lindley (GL) distribution.

\begin{tabular}{|ccccc|}
\hline Data & Model & $\mu$ & $\beta$ & $\gamma$ \\
\hline \multirow{3}{*}{ data 1 } & L & $0.0112(0.0009)$ & & \\
& GL & $0.0175(0.0030)$ & $2.1052(0.4872)$ & \\
& GL & $0.0182(0.0034)$ & $2.3367(0.7700)$ & $0.1474(0.4428)$ \\
\hline \multirow{3}{*}{ data 2 } & L & $0.2572(0.0239)$ & & \\
& WL & $2.6100(0.4796)$ & $17.3488(3.3002)$ & \\
& GL & $2.6242(0.4949)$ & $17.6186(4.4834)$ & $6.0704(62.5868)$ \\
\hline
\end{tabular}

In Table 2 are listed standard model selection measures: $-2 \times \log$-likelihood, AIC (Akaike's Information Criterion, [1]) and BIC (Schwarz's Bayesian Information Criterion, [34]). From the values of these statistics we conclude that the two parameter Lindley distribution provides a better fit for the data sets when compared to the two alternative models. For the WL model the obtained estimates for $\theta$ are respectively given by: 176.82 (data set 1) and 6.98 (data set 2). The standard errors are given by, 11.86 and 0.21 , respectively.

For illustrative purposes, we present in Figure 1 the 50\%, 90\% and 95\% likelihood contour plots in the original and the proposed orthogonal parametrization. In contrast to panels ( $b$, data set 1$)$ and ( $d$, data set 2$)$, the orientation of the contours in panels ( $a$, data set 1$)$ and (c, data set 2$)$ revels a high positive correlation between $\widehat{\beta}$ and $\widehat{\mu}$. We observe in panels $(b)$ and $(d)$ that the 
Table 2 - Model selection measures.

\begin{tabular}{|ccccc|}
\hline Data & Model & -2 log-like & AIC & BIC \\
\hline \multirow{4}{*}{ Data 1 } & L & 858.6 & 860.6 & 862.8 \\
& WL & 851.5 & 855.5 & 860.1 \\
& GL & 851.3 & 857.3 & 864.2 \\
\hline \multirow{3}{*}{ Data 2 } & L & 316.7 & 318.7 & 320.8 \\
& WL & 223.6 & 227.6 & 231.8 \\
& GL & 223.6 & 229.6 & 235.9 \\
\hline
\end{tabular}

axes of the elliptical contours are parallel to the coordinate axes, and for this reason we have an indication that the correlation is equal to zero. Naturally, this is expected since $\theta$ and $\beta$ are estimated independently. These contours were built using the procedure described in [16] and also presented in [27].

In Table 3 we present, for both data sets, the corresponding p-values to Kolmogorov-Smirnov (K-S) and Anderson-Darling (A-D) goodness-of-fit statistics. From these results, it is clear that the WL distribution provides a good fit to the given data sets. We also consider the Log-Normal distribution (LN) in the data analysis, since this distribution was considered by [14] (data set 1) and by [25] (data-set 2).

Table 3 - Kolmogorov-Smirnov and Anderson-Darling goodness-of-fit statistics.

\begin{tabular}{|ccccc|}
\hline \multicolumn{5}{|c|}{ data set 1 } \\
\hline Test & L & WL & GL & LN \\
\hline K-S & 0.0274 & 0.5681 & $<0.0001$ & 0.3200 \\
A-D & 0.1037 & 0.6668 & $<0.0001$ & 0.5274 \\
\hline \multicolumn{5}{|c|}{ data set 2 } \\
\hline Test & L & WL & GL & LN \\
\hline K-S & $<0.0001$ & 0.9085 & $<0.0001$ & 0.7335 \\
A-D & $<0.0001$ & 0.9776 & $<0.0001$ & 0.8744 \\
\hline
\end{tabular}

\section{CONCLUDING REMARKS}

In this paper we introduced an alternative parametrization for the shape parameter of the weighted Lindley distribution (WL) introduced by [12], which generalizes the one parameter Lindley distribution. In the proposed parametrization, the new parameter have a direct interpretation and it is orthogonal to the shape parameter.

In the last years, the Lindley distribution have been considered in several applications as an alternative lifetime model and its generalization called weighted Lindley distribution considering the orthogonal parametrization could be another good alternative distribution to modeling lifetime data. We fitted the WL distribution to two real data sets and compared the obtained results with those of $\mathrm{L}$ and GL distributions, which showed the great potentialities of the WL distribution. 


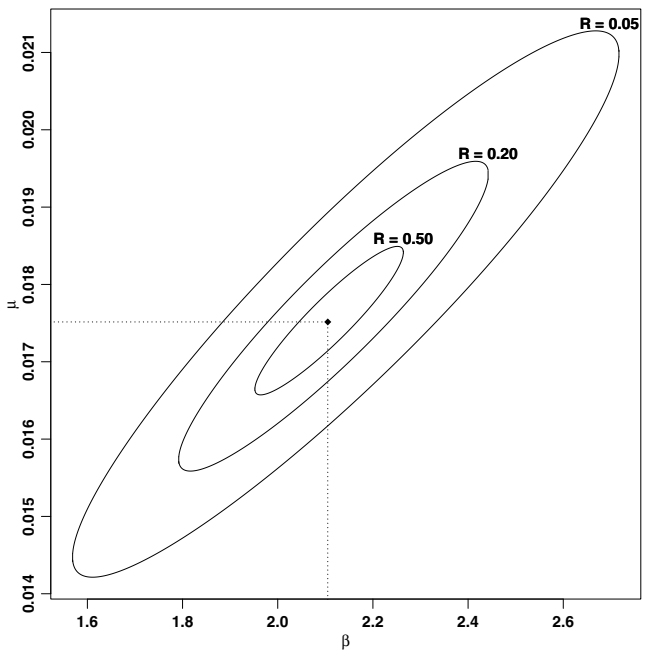

(a)

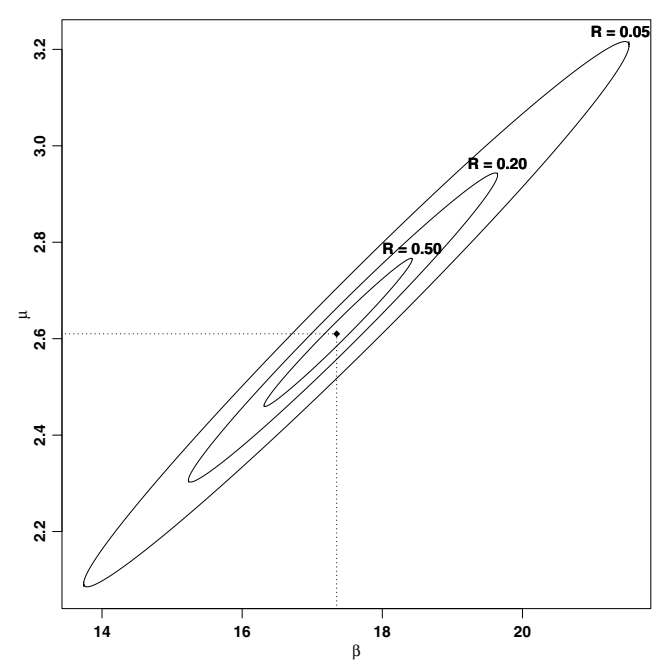

(c)

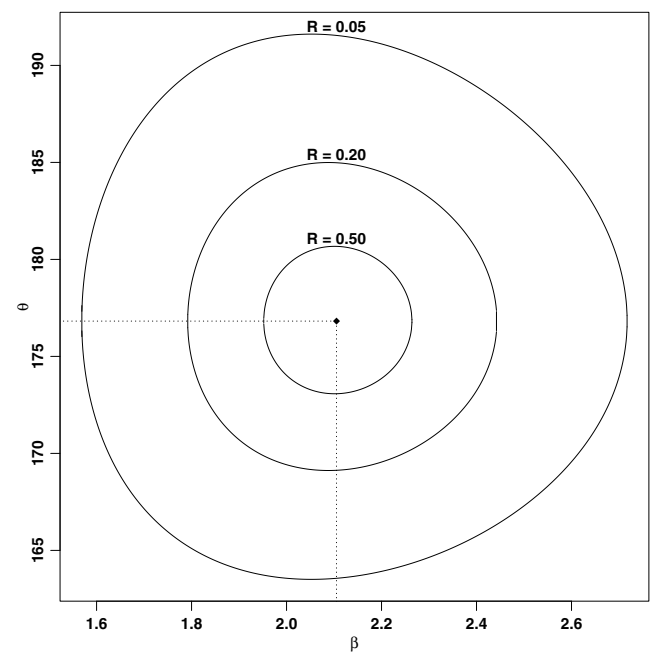

(b)

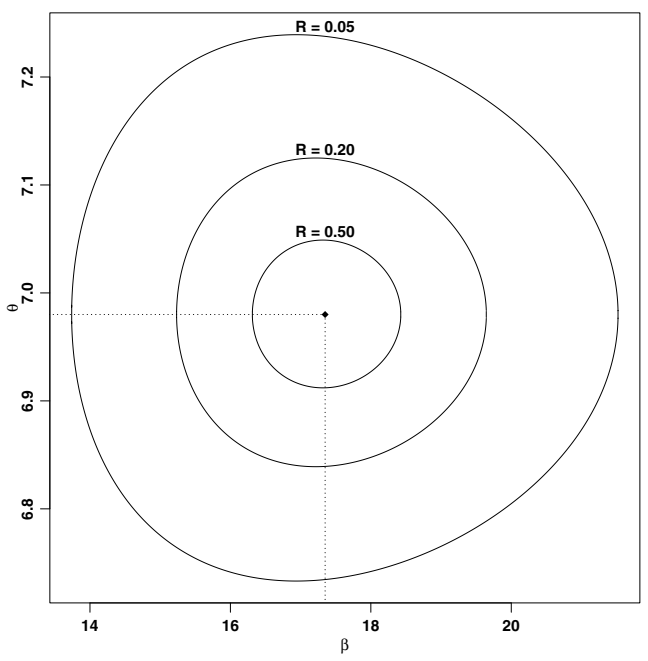

(d)

Figure $1-(a, c)$ : Contour plot for the joint relative likelihood function of $\beta$ and $\mu .(b, d)$ : Contour plot for the joint relative likelihood function of $\beta$ and $\theta$. 


\section{REFERENCES}

[1] Akaike H. 1983. Information measures and model selection. In: 'Proceedings of the 44th session of the International Statistical Institute, Vol. 1 (Madrid, 1983)', Vol. 50, pp. 277-290. With a discussion in Vol. 3, pp. 209-219.

[2] BARndorfF-Nielsen OE \& Cox DR. 1994. Inference and asymptotics, Vol. 52 of Monographs on Statistics and Applied Probability, Chapman \& Hall, London.

[3] Bebbington M, Lai C \& Zitikis R. 2007. Bathtub-type curves in reliability and beyond. Australian \& New Zealand Journal of Statistics, 49(3): 251-265.

[4] BJERKEDAL T. 1960. Acquisition of resistance in guinea pigs infected with different doses of virulent tubercle bacilii. Amer. J. Hyg., 72: 130-148.

[5] Borah M \& Begum RA. 2002. Some properties of Poisson-Lindley and its derived distributions. Journal of the Indian Statistical Association, 40(1): 13-25.

[6] Borah M \& Deka Nath A. 2001. Poisson-Lindley and some of its mixture distributions. Pure and Applied Mathematika Sciences, 53(1-2): 1-8.

[7] Borah M \& Deka Nath A. 2001. A study on the inflated Poisson Lindley distribution. Journal of the Indian Society of Agricultural Statistics, 54(3): 317-323.

[8] CHEN Z. 2000. A new two-parameter lifetime distribution with bathtub shape or increasing failure rate function. Statistics \& Probability Letters, 49: 155-161.

[9] Cox DR \& REID N. 1987. Parameter orthogonality and approximate conditional inference. Journal of the Royal Statistical Society. Series B, 49(1): 1-39. With a discussion.

[10] Ghitany ME, Al-Mutairi DK \& Nadarajah S. 2008. Zero-truncated Poisson-Lindley distribution and its application. Mathematics and Computers in Simulation, 79(3): 279-287.

[11] Ghitany ME \& AL-Mutari DK. 2008. Size-biased Poisson-Lindley distribution and its application. METRON - International Journal of Statistics, 66(3): 299-311.

[12] Ghitany ME, Alqallaf F, Al-Mutairi DK \& Husain HA. 2011. A two-parameter weighted Lindley distribution and its applications to survival data', Mathematics and Computers in Simulation, 81: 1190-1201.

[13] Ghitany ME, Atieh B \& Nadarajah S. 2008. Lindley distribution and its application. Mathematics and Computers in Simulation, 78(4): 493-506.

[14] Gupta RC, Kannan N \& Raychaudhuri A. 1997. Analysis of Lognormal survival data. Mathematical Biosciences, 139: 103-105.

[15] HAUPT E \& SCHÄBE H. 1992. A new model for a lifetime distribution with bathtub shaped failure rate. Microelectronics Reliability, 32(5): 33-639.

[16] Kalbfleisch JG. 1985. Probability and statistical inference. Vol. 2, Springer Texts in Statistics, second edn, Springer-Verlag, New York.

[17] Lai CD, Xie M \& Murthy DNP. 2001. Bathtub-shaped failure rate life distributions. In: 'Advances in reliability', Vol. 20 of Handbook of Statistics. North-Holland, Amsterdam, pp. 69-104.

[18] LAN Y \& LeEMIS LM. 2008. The logistic-exponential survival distribution. Naval Res. Logist., 55(3): 252-264. 
[19] LAnCASter T. 2002. Orthogonal parameters and panel data. Review of Economic Studies, 69(3): 647-666.

[20] Lindley D. 1965. Introduction to Probability and Statistics from a Bayesian Viewpoint, Part II: Inference. Cambridge University Press, New York.

[21] Lindley DV. 1958. Fiducial distributions and Bayes' theorem. Journal of the Royal Statistical Society. Series B. Methodological, 20: 102-107.

[22] Louzada F \& CAVAli W. 2014. On a double reparametrization for accelerated lifetime testing. Chilean Journal of Statistics, 5(1): 37-48.

[23] Louzada-Neto F \& Pardo-Fernandez. 2001. The effect of reparametrization on the accuracy of inferences for accelerated lifetime tests. Journal of Applied Statistics, 28: 703-711.

[24] Mahmoudi E \& Zakerzadeh H. 2010. Generalized Poisson Lindley distribution. Communications in Statistics Theory and Methods, 39: 1785-1798.

[25] Martín J \& PÉRez CJ. 2009. Bayesian analysis of a generalized lognormal distribution. Computational Statistics \& Data Analysis, 53: 1377-1387.

[26] Mazucheli J \& Achcar JA. 2011. The Lindley Distribution Applied to Competing Risks Lifetime Data. Computer Methods and Programs in Biomedicine, 104(2): 188-192.

[27] Meeker WQ \& Escobar LA. 1998. Statistical Methods for Reliability Data. John Wiley \& Sons, New York.

[28] NADARAJAH S. 2008. Bathtub-shaped failure rate functions. Quality \& Quantity, 43(5): 855-863.

[29] Olver FWJ, Lozier DW, Boisvert RF \& ClARK CW. (eds.) 2010. NIST Handbook of mathematical functions, U.S. Department of Commerce National Institute of Standards and Technology, Washington, DC.

[30] Rajarshi S \& RAJARshi MB. 1988. Bathtub distributions: A review. Communications in Statistics. Theory and Methods, 17: 2597-2621.

[31] Sankaran M. 1970. The discrete Poisson-Lindley distribution. Biometrics, 26: 145-149.

[32] SAS. 2010. The NLMIXED Procedure, SAS/STAT ${ }^{\circledR}$ User's Guide, Version 9.22, Cary, NC: SAS Institute Inc.

[33] Schafft HA, Staton TC, Mandel J \& Shott JD. 1987. Reproducibility of electromigration measurements. IEEE Transactions on Electronic Device, 34(3): 673-681.

[34] SCHWARZ GE. 1978. Estimating the dimension of a model. Annals of Statistics, 6(2): 461-464.

[35] Smith RM \& Bain LJ. 1975. An Exponential Power Life-Test Distribution. Communications in Statistics, 4(5): 469-481.

[36] Zakerzadeh H \& Dolati A. 2009. Generalized Lindley distribution. Journal of Mathematical Extension, 3(4): 13-25.

[37] Zamani H \& Ismail N. 2010. Negative Binomial-Lindley distribution and its application. Journal of Mathematics and Statistics, 6(1): 4-9. 